\title{
Detection of Changes in Renal Blood Flow Using Arterial Spin Labeling MRI
}

\author{
Dennis Kannenkeril ${ }^{a}$ Rolf Janka ${ }^{b}$ Agnes Bosch $^{a}$ Susanne Jung ${ }^{a, c}$ \\ Julie Kolwelter ${ }^{a, c}$ Kristina Striepe ${ }^{a}$ Christian Ott $^{a}$ Petros Martirosian $^{d}$ \\ Mario Schiffer ${ }^{\mathrm{a}}$ Michael Uder $^{\mathrm{b}}$ Roland E. Schmieder ${ }^{\mathrm{a}}$
}

aDepartment of Nephrology and Hypertension, University Hospital Erlangen, Erlangen, Germany; ${ }^{\mathrm{b}}$ Institute of Radiology, University Hospital Erlangen, Erlangen, Germany; 'Department of Cardiology, University Hospital Erlangen, Erlangen, Germany; ${ }^{d}$ Section on Experimental Radiology, University Hospital of Tübingen, Tübingen, Germany

\section{Keywords}

ASL-MRI $\cdot$ Renal blood flow $\cdot$ Cold pressor test

\begin{abstract}
Background: Alteration in kidney perfusion is an early marker of renal damage. The purpose of this study was to evaluate if changes in renal blood flow (RBF) could be detected using MRI with arterial spin labeling (ASL) technique. Methods: RBF as assessed by cortical (CRBF), medullary, and total renal blood flow (TRBF) were measured by MRI with arterial spin labeling (ASL-MRI) using flow-sensitive alternating inversion recovery true fast imaging with steady-state precession sequence. In 11 normotensive healthy individuals (NT) and 11 hypertensive patients (HT), RBF was measured at baseline and after both feet were covered with cold ice packs (cold pressor test) that activates the sympathetic nervous system. In another experiment, RBF was measured in 10 patients with CKD before and after a pharmacological intervention. We compared RBF measurements between the 3 study populations. Results: A significant reduction in $\operatorname{CRBF}(p=0.042)$ and a trend in TRBF ( $p=0.053)$ were observed in response to the activation of the sympathetic nervous system. A trend toward reduction of CRBF $(p=0.051)$ and TRBF $(p=0.059)$
\end{abstract}

has been detected after pharmacological intervention. TRBF was significantly lower in patients with $\mathrm{HT}$ and CKD patients compared to NT individuals (NT vs. HT, $p=0.014$; NT vs. CKD, $p=0.004)$. TRBF was lower in patients with CKD compared to HT $(p=0.047)$. Conclusion: Our data indicate that both acute and short-term changes in RBF could be detected using ASLMRI. We were able to detect differences in RBF between healthy and diseased individuals by needing only small sample size per group. Thus, ASL-MRI offers an advantage in conducting clinical trials compared to other technologies.

(c) 2021 S. Karger AG, Basel

\section{Introduction}

The prevalence of CKD is $8-16 \%$ worldwide $[1,2]$ and represents an important, severe, and independent risk factor of cardiovascular disease, even in its early stage [3]. The risk of patients with CKD to develop ESRD is less likely compared to the risk to die resulting from cardiovascular disease [4]. Early identification of CKD is needed to prevent disease progression toward ESRD and reduce the risk of cardiovascular morbidity and mortality. karger@karger.com

www.karger.com/ajn

Karger
(C) 2021 S. Karger AG, Basel

to

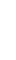

Roland E. Schmieder

Department of Nephrology and Hypertension, Head of the Clinical Research Center University Hospital Erlangen

Ulmenweg 18, DE-91054 Erlangen (Germany)

roland.schmieder@uk-erlangen.de 
In early stages of diseases like diabetes mellitus and arterial hypertension, renal autoregulatory mechanisms try to keep renal blood flow (RBF) constant and tissue injury at minimum [5]. Autoregulation is mainly based on the myogenic response of smooth muscle in response to external stretching force and on tubuloglomerular feedback that leads to vasoconstriction of the afferent arteriole in response to an increased sodium concentration in the early distal tubule [6]. For example, damage of renal microvasculature due to high blood pressure (BP) is prevented by proportionate autoregulatory vasoconstriction of the preglomerular vasculature thereby keeping glomerular hydrostatic pressures constant. Long-term increase in BP leads to impairment of the renal autoregulation, resulting in renal hyperperfusion and hyperfiltration in the early stage and CKD develops consecutively [7].

Definition and classification of CKD is based on estimation of glomerular filtration rate (eGFR) and measurement of albuminuria [1]. However, alteration in RBF occurs earlier and represents an early marker of renal alteration in hypertensive and diabetic CKD. Measurement of RBF may help to identify early stages of CKD and therefore may allow to intervene and stop the progression of CKD at an early stage. Functional MRI with arterial spin labeling (ASL-MRI) is a noninvasive method for quantification of RBF without the use of contrast media. Its clinical utility is yet under investigation $[8,9]$.

In this study our goal was to analyze the applicability of ASL-MRI technique in 2 experiments assessing both acute and short-term changes in RBF. Our hypothesis was that first, ASL-MRI is able to detect acute as well as short-term changes in RBF induced by a physiological and/or pharmacological intervention and second that ASL-MRI is able to detect differences in RBF between study populations by using small study cohorts.

\section{Methods}

\section{Study Design}

This study consisted of 2 clinical experiments which are explained in detail below. The study population consisted of normotensive healthy individuals (NT), patients with primary arterial hypertension (HT) and patients with CKD stage 3. All individuals participated in clinical trials performed at the Clinical Research Unit of the Department of Nephrology and Hypertension, University of Erlangen, Germany (www.clinicaltrials.gov: NCT02746549). The study protocol was approved by the Ethics Committee of the University Erlangen and performed in accordance with the Declaration of Helsinki and the principles of Good Clinical Practice guidelines. Individuals were recruited by advertising in local news-
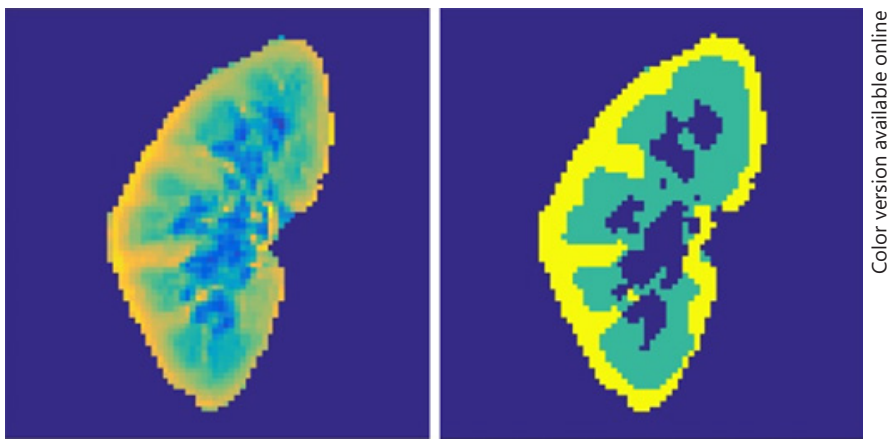

Fig. 1. Illustration of perfusion images of the right kidney with differentiation of cortical (yellow) and medullary perfusion (green). TRBF, CRBF, and MRBF were assessed separately using a cortexmedulla segmentation step. TRBF, total renal blood flow; CRBF, cortical renal blood flow; MRBF, medullary renal blood flow.

papers in the area of Erlangen-Nürnberg, Germany, or by referral of primary care physician to our outpatient clinic. All patients provided written informed consent prior to inclusion in the study.

\section{Study Population}

Individuals of NT group had normal BP, no significant disease or any significant laboratory abnormality, and had no medication that might influence renal perfusion. Patients with uncomplicated primary HT with mean sitting systolic BP $\geq 140 \mathrm{~mm} \mathrm{Hg}$ and/or diastolic $\mathrm{BP} \geq 90 \mathrm{~mm} \mathrm{Hg}$ or on antihypertensive medications were included in the HT group. Patients with stable moderately impaired renal function, defined as an eGFR $30-59 \mathrm{~mL} / \mathrm{min} / 1.73 \mathrm{~m}^{2}$, calculated using the 4 -variable Modification of Diet in Renal Disease (MDRD) Study equation were included in the CKD group. Office BP was measured in a standardized fashion according to guideline recommendations [10]. Patients with severe hypertension (systolic BP $\geq 180 \mathrm{~mm} \mathrm{Hg}$ and/or diastolic BP $\geq 100 \mathrm{~mm} \mathrm{Hg}$ ) were excluded from the study. Patients with any other serious disease were also excluded.

\section{Arterial Spin Labeling MRI}

ASL-MRI technique combined with semiautomatic segmentation for differential cortical (CRBF) and medullary renal blood flow (MRBF) assessment and analysis of the images obtained using this technique have been described in detail in our former studies $[11,12]$. ASL-MRI in this study was performed with a $1.5 \mathrm{~T}$ MRI (Magnetom Aera, Siemens Healthineers, Erlangen, Germany) using a flow-sensitive alternating inversion recovery (FAIR) true fast imaging with steady-state precession (True-FISP) sequence, which combines a FAIR perfusion preparation and a True-FISP data acquisition strategy. The FAIR True-FISP parameters included the following: repetition time, $4.0 \mathrm{~ms}$; echo time, $2.0 \mathrm{~ms}$; effective inversion time, 1,200 ms; flip angle, $70^{\circ}$; field of view, $400 \mathrm{~mm}$; slice thickness, $5 \mathrm{~mm}$; and in-plane resolution, $1.6 \times 1.6 \mathrm{~mm}$.

A software allowed us to assess total renal blood flow (TRBF), $\mathrm{CRBF}$, and MRBF separately using a cortex-medulla segmentation step (Fig. 1). Eight pairs of global/slice inversion images and $1 \mathrm{M} 0$ image were acquired. To overcome fluctuations in the breath-hold results, 1 image has to be segmented semiautomatically. The re- 
Table 1. Clinical characteristics and ASL-MRI data of study populations

\begin{tabular}{lccc}
\hline Parameter & NT $(N=11)$ & HT $(N=11)$ & CKD $(N=10)$ \\
\hline Age, years & $35.2 \pm 12.0$ & $39.2 \pm 10.3$ & $68.3 \pm 7.8$ \\
Gender (male/female) & $11 / 0$ & $11 / 0$ & $8 / 2$ \\
Body weight, kg & $78.0 \pm 7.5$ & $93.4 \pm 14.9$ & $92.7 \pm 18.3$ \\
BMI, $\mathrm{kg} / \mathrm{m}^{2}$ & $23.0 \pm 2.3$ & $28.3 \pm 3.4$ & $30.7 \pm 5.0$ \\
Office SBP, mm Hg & $131.5 \pm 8.5$ & $133.8 \pm 10.3$ & $131.3 \pm 11.9$ \\
Office DBP, mm Hg & $80.0 \pm 7.7$ & $82.8 \pm 8.0$ & $74.2 \pm 9.0$ \\
Office HR, bpm & $69.1 \pm 11.4$ & $67.9 \pm 8.1$ & $69.9 \pm 11.1$ \\
Serum sodium, mmol/L & $140.0 \pm 1.7$ & $140.7 \pm 1.5$ & $141.3 \pm 1.8$ \\
Serum potassium, mmol/L & $4.3 \pm 0.4$ & $4.3 \pm 0.3$ & $5.0 \pm 0.6$ \\
Serum Cr, mg/dL & $0.89 \pm 0.10$ & $0.90 \pm 0.13$ & $1.7 \pm 0.4$ \\
eGFR, mL/min per $1.73 \mathrm{~m}{ }^{2}$ & $108.0 \pm 14.0$ & $105.1 \pm 15.2$ & $41.9 \pm 10.7$ \\
HbAlc, $\%$ & $5.3 \pm 0.2$ & $5.3 \pm 0.4$ & - \\
Triglyceride, mg/dL & $116.5 \pm 90.1$ & $129.0 \pm 79.5$ & $171.2 \pm 85.1$ \\
Total cholesterol, mg/dL & $202.7 \pm 56.5$ & $213.1 \pm 42.6$ & $233.9 \pm 133.7$ \\
LDL, mg/dL & $133.6 \pm 41.4$ & $141.2 \pm 33.3$ & - \\
HDL, mg/dL & $49.7 \pm 9.6$ & $51.6 \pm 10.1$ & - \\
TRBF, mL/100 g/min & $338.7 \pm 30.0$ & $309.5 \pm 17.3$ & $260.4 \pm 29.1$ \\
CRBF, mL/100 g/min & $387.6 \pm 41.1$ & $349.0 \pm 33.3$ & $286.4 \pm 33.1$ \\
MRBF, mL/100 g/min & $314.8 \pm 39.7$ & $288.5 \pm 15.8$ & $234.6 \pm 35.3$ \\
\hline
\end{tabular}

Data are given as mean \pm SD. ASL-MRI, MRI with arterial spin labeling; NT, normotensive healthy individuals; HT, patients with hypertension; SBP, systolic blood pressure; DBP, diastolic blood pressure; HR, heart rate; BPM, beats per minute; eGFR, estimated glomerular filtration rate calculated using CKD-epi formula; LDL, low density lipids; HDL, high density lipids; TRBF, total renal blood flow; CRBF, cortical renal blood flow; MRBF, medullary renal blood flow.

sulting segmentation is registered onto the remaining 7 pairs of global/slice inversion images using a stiff registration. Image pairs which do not fit into the segmented contour will be sorted out. With the remaining image pairs, TRBF is automatically calculated by the software using the formula described previously [11]. To distinct cortex and medulla perfusion, the gray values of medulla, cortex, calyces, and background were separated on the registered global inversion images using the k-means clustering method [13]. For increased robustness, we extended this gray value-based approach by adding the distance to the kidney's center of gravity into the computation. Since we were especially interested in correct cortical perfusion values, a morphological filter was used to ensure that no medullar or background pixels remain in the region of the segmented cortical area. Although this may lead to a slightly undersegmented cortex, the segmentation process guarantees to only contain pixels completely belonging to the cortex.

\section{Experiment 1}

This experiment was carried out in individuals of NT group and in patients with HT. After initial ASL-MRI measurements, both feet were covered with ice packs at $1^{\circ} \mathrm{C}$, which triggers sympathetic activation, leading to vasoconstriction in the systemic circulation (cold pressor test). Then ASL-MRI measurements were repeated. During both MRI measurements, $\mathrm{BP}$ and heart rate (HR) were measured continuously every $2 \mathrm{~min}$.

\section{Experiment 2}

In the second experiment, after baseline ASL-MRI measurements, patients with nondiabetic CKD underwent pharmacologic intervention of a potentially nephroprotective drug (RAS inhibition combined with neprilysin inhibition) under investigation. On the 7th day of intervention, ASL-MRI measurements were repeated.

\section{Evaluation of RBF between 3 Different Study Populations}

TRBF, CRBF, and MRBF were compared by using ASL-MRI in 3 different study populations (healthy NT individuals, patients with $\mathrm{HT}$, and patients with $\mathrm{CKD}$ ). We analyzed also whether the various RBF parameters assessed by ASL-MRI were related to clinical characteristics.

\section{Statistics}

Normal distribution of data was confirmed by KolmogorovSmirnov tests and histograms before further analysis. Normally distributed data were compared by paired and unpaired Student's $t$ test. For non-normally distributed parameters, Mann-Whitney $\mathrm{U}$ test was used for unpaired and Wilcoxon test for paired analysis, respectively. Data are presented as means with standard deviation (SD) and percentages, respectively. Bivariate correlation analyses were performed using Pearson's test for normally distributed data and Spearman's test for non-normally distributed data. Two-tailed values of $p<0.05$ were considered statistically significant, and we report the precise $p$ values. Statistical analysis was performed using IBM SPSS Statistics 21.0.0.2, Philadelphia, PA, USA. 
Fig. 2. a Change in CRBF following a physiological stress (cold pressor test) in NT individuals and patients with HT. The image box plots represent CRBF before (CRBF baseline) and during application of $1^{\circ} \mathrm{C}$ ice packs (CRBF ice). $\mathbf{b}$ Change in CRBF following a pharmacological intervention in patients with CKD. The image box plots represent $\mathrm{CRBF}$ before (CRBF baseline) and after pharmacological intervention (CRBF pharmacological intervention). c Comparison of TRBF between 3 study populations. CRBF, cortical renal blood flow; TRBF, total renal blood flow; NT, normotensive healthy individuals; HT, patients with hypertension. $p$ values were adjusted for age and gender, where applicable.

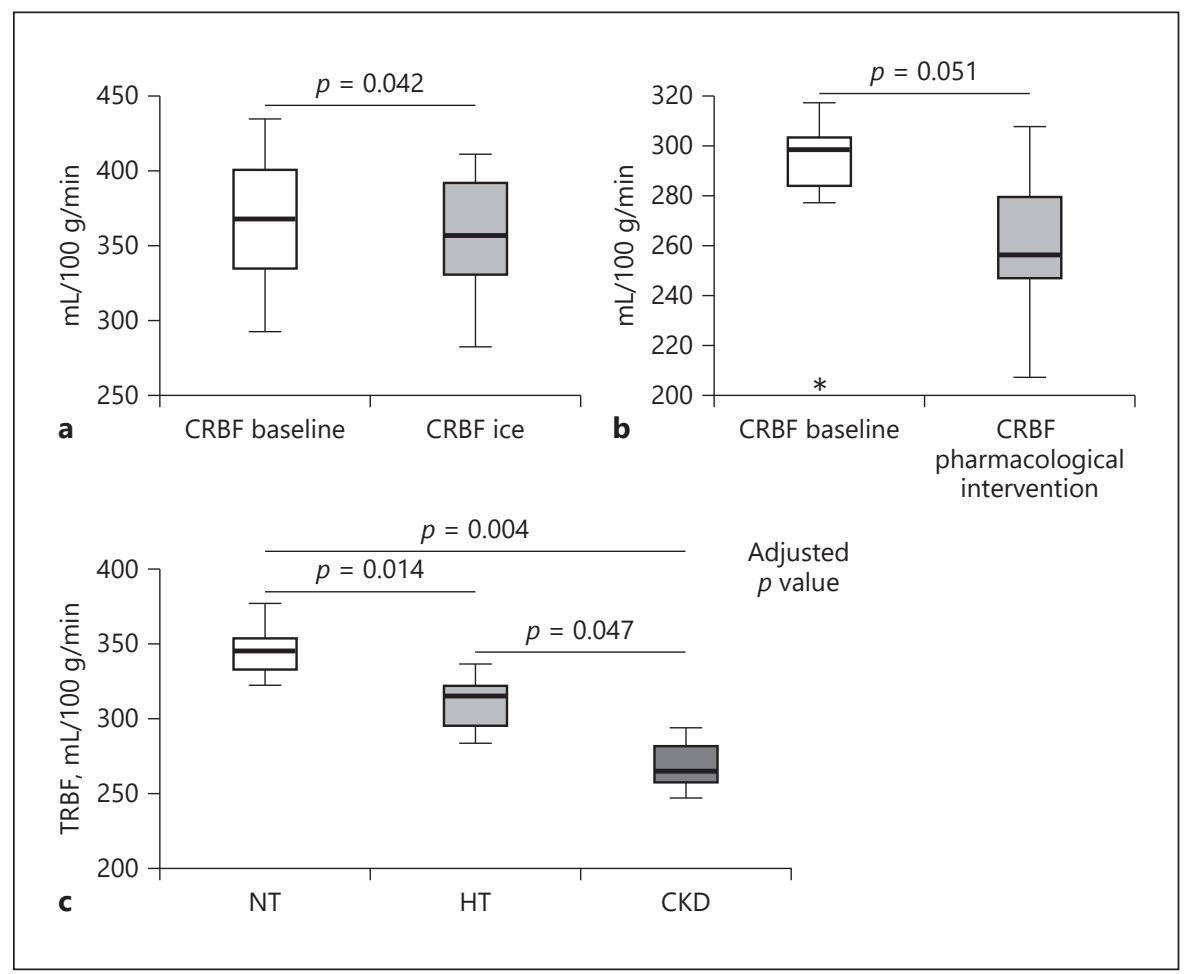

\section{Results}

\section{Study Population}

In experiment 1, 11 male NT individuals and 11 male patients with HT were analyzed. Mean duration of HT in these 11 individuals was $10.4 \pm 7.5$ years. They were treated with an average of $1.7 \pm 0.8$ in these 11 indiv medications. Mean urinary albumin Cr ratio was not significantly different between NT individuals and patients with HT. Experiment 2 was conducted in 8 male and 2 female patients with CKD (mean eGFR of $41.9 \pm 10.7 \mathrm{~mL} / \mathrm{min}$ per $\left.1.73 \mathrm{~m}^{2}\right)$. Patients with CKD were older $(68.3 \pm 7.8$ years $)$ compared to individuals of the other 2 groups (NT individuals $35.2 \pm 12$ years, and patients with HT $39.2 \pm 10.3$ years, respectively). All individuals from experiment 1 $(n=22)$ and $2(n=10)$ were included in the evaluation of RBF in 3 different study populations. The clinical characteristics of the study populations are given in Table 1.

\section{Experiment 1 - Change in RBF following a}

\section{Physiological Stress}

A hemodynamic response with respect to systolic BP, diastolic BP, and HR has been provoked immediately after covering both feet with ice packs. Both systolic (131.1 \pm 16.2 vs. $141.4 \pm 15.2 \mathrm{~mm} \mathrm{Hg} ; p<0.001)$ and diastolic BP (72.8 \pm 11.8 vs. $82.9 \pm 9.2 \mathrm{~mm} \mathrm{Hg} ; p<0.001)$ and HR
$(68.1 \pm 8.2$ vs. $74.2 \pm 12.0 \mathrm{bpm} ; p=0.002)$ increased significantly during sympathetic activation. In parallel, we found a significant reduction in CRBF $(368.3 \pm 41.5$ vs. $357.6 \pm 38.5 \mathrm{~mL} / 100 \mathrm{~g} / \mathrm{min} ; p=0.042$; Fig. $2 \mathrm{a}$ ) in response to the cold pressor test. A trend has been noticed with respect to the TRBF $(324.1 \pm 28.2$ vs. $318.7 \pm 27.8 \mathrm{~mL} / 100$ $\mathrm{g} / \mathrm{min} ; p=0.053)$. No change in MRBF has been noticed (301.7 \pm 32.4 vs. $297.5 \pm 32.6 \mathrm{~mL} / 100 \mathrm{~g} / \mathrm{min} ; p=0.592)$.

\section{Experiment 2 - Change in RBF with Pharmacological} Intervention

In the second experiment, a significant reduction in systolic BP (131.3 \pm 11.9 vs. $119.1 \pm 9.4 \mathrm{~mm} \mathrm{Hg} ; p=0.002)$ has been noticed after 7 days of pharmacological intervention. No change has been found with respect to diastolic BP (74.2 \pm 9.0 vs. $70.2 \pm 6.9 \mathrm{~mm} \mathrm{Hg} ; p=0.060)$ and $\operatorname{HR}(69.9 \pm 11.1$ vs. $73.2 \pm 10.4 \mathrm{~mm} \mathrm{Hg} ; p=0.211)$. A trend of reduction in CRBF $(286.4 \pm 33.1$ vs. $263.0 \pm 32.0$ $\mathrm{mL} / 100 \mathrm{~g} / \mathrm{min} ; p=0.051$; Fig. $2 \mathrm{~b})$ and in TRBF $(260.4 \pm$ 29.1 vs. $250.0 \pm 24.2 \mathrm{~mL} / 100 \mathrm{~g} / \mathrm{min} ; p=0.059)$ was detected. No change in MRBF was found (234.6 \pm 35.3 vs. $225.7 \pm 30.3 \mathrm{~mL} / 100 \mathrm{~g} / \mathrm{min} ; p=0.327$ ).

Comparison of RBF between 3 Different Study Cohorts

TRBF in patients with HT $(309.5 \pm 17.3 \mathrm{~mL} / 100 \mathrm{~g} /$ $\mathrm{min})$ and patients with CKD $(260.4 \pm 29.1 \mathrm{~mL} / 100 \mathrm{~g} / \mathrm{min})$ 
were significantly lower compared to NT individuals $(338.7 \pm 30.0 \mathrm{~mL} / 100 \mathrm{~g} / \mathrm{min})$. When potential confounders such as age and gender were entered in the covariance analysis, TRBF was still found to be higher in NT individuals than in other groups (NT vs. HT - age adjusted $p=0.014$, NT vs. CKD - age and gender adjusted $p=$ 0.004; Fig. 2c). TRBF was also higher in patients with HT than in patients with CKD (age and gender adjusted $p=$ 0.047; Fig. 2c). Similar results have been found with respect to CRBF but not with regard to MRBF (Table 1).

\section{Relation between RBF and Clinical Parameters}

We found a significant correlation between TRBF assessed by using the ASL-MRI technique and clinical characteristics of all study participants combined $(n=32)$ with respect to age $(r=-0.650, p<0.001)$, BMI $(r=$ $-0.720, p<0.001)$ and serum $\operatorname{Cr}(r=-0.726, p<0.001)$. Similar correlations have been found with CRBF (age: $r=$ $-0.548, p=0.001$; BMI: $r=-0.561, p=0.001$; serum Cr: $r=-0.700, p<0.001$ ) and MRBF (age: $r=-0.654, p<$ 0.001; BMI: $r=-0.621, p<0.001$; serum Cr: $r=-0.723$, $p<0.001)$.

\section{Discussion}

Change in renal perfusion is one of the early markers of renal damage [2]. Previously, we have demonstrated that the ASL-MRI technique is a reliable tool to detect RBF with low variability of total, cortical, and medullary $\mathrm{RBF}$ [12]. The main finding of the present study is that both acute and short-term changes in RBF can be analyzed using the ASL-MRI technique with small sample sizes of individuals needed to be included in order to detect significant changes after physiological or pharmacological provocations. Moreover, only a small sample size was needed to detect the expected difference in CRBF and TRBF between healthy individuals and diseased individuals with either HT or CKD.

One of the advantages of ASL-MRI is that the sequence and scanning protocol enabled us to perform 8 repetitive scans in approximately $5 \mathrm{~min}$. This short measurement duration allows further dynamic renal perfusion measurement during acute interventions. Thus, ASL-MRI provides a distinct advantage over para-amiohippuric acid (PAH) clearance [14] and dynamic contrast-enhanced MRI [15], which are not repeatable within such a short time frame. We investigated the response of RBF in both the renal cortex and medulla of humans during a physiologically induced acute stimulation of the sympa-

Change in Renal Blood Flow \& Arterial Spin Labeling MRI thetic nervous system. Increased sympathetic nerve activity to the kidneys causes a reduction of the RBF and GFR and reduction of sodium and water excretion [16]. In response to the sympathetic activation, an acute reduction in CRBF, a trend toward lower TRBF, without any change in MRBF have been observed in NT individuals and patients with $\mathrm{HT}$, in parallel to significant increase in $\mathrm{BP}$ and HR. In another study using handgrip exercise instead of ice provocation as a sympathetic trigger, significant decreases in both the cortical and medullary compartment were noted [17]. In accordance to our finding, studies in animals have demonstrated an insensitivity of MRBF to change in response to renal sympathetic nerve stimulation [18].

In our second experiment, 10 patients with stage 3 CKD received a pharmacological intervention of a potentially nephroprotective drug for 7 days. After initial analysis of RBF using the ASL-MRI technique, all patients received a second ASL-MRI 7 days after starting the pharmacologic intervention. We found a trend toward reduction in TRBF and CRBF. Previously, we demonstrated that significant changes in renal perfusion in individuals with metabolic syndrome in response to a 2 -week treatment period with an angiotensin receptor blocker could be detected by the ASL-MRI technique and that these changes were related to the changes of renal plasma flow measured in parallel by PAH plasma clearance [11]. In a subsequent study, we reported in accordance a significant increase in renal perfusion after renin inhibition in patients with HT, using ASL-MRI [19]. Our second experiment indicates that changes in renal perfusion can be assessed by using ASL-MRI in small sample sizes which makes ASL-MRI attractive to be applied in clinical trials. Furthermore, our results suggest that ASL-MRI is a promising tool for the separate assessment of cortical and medullary RBF.

We compared the RBF between the 3 different study populations. Compared to healthy NT individuals, TRBF was lower in patients with HT and patients with CKD, and a significant difference in TRBF was found between patients with HT and CKD. Noticeably, the number of individuals we needed per study group to measure a significant difference in TRBF was $<12$ individuals which is explained by the low variability of ASL-MRI measurements, with approximate $\mathrm{SD}$ of $30 \mathrm{~mL} / 100 \mathrm{~g} / \mathrm{min}$ and less in each population. For comparison, the SD of renal plasma flow measured using PAH steady-state clearance technique has been previously described to be approximately $125 \mathrm{~mL} / \mathrm{min} \mathrm{[20],} \mathrm{in} \mathrm{other} \mathrm{words} 4$ times more than with the MRI ASL technique. Accordingly, we could 
not find a significant difference in renal plasma flow using the clearance technique between NT individuals and patients with HT [21].

Finally, we analyzed the relationship between TRBF assessed using ASL-MRI and clinical characteristics. We found a significant association of TRBF with age, BMI, and serum Cr. Similar correlations have been found with above-mentioned parameters have been formerly described and are well known $[22,23]$.

Our study has strengths and limitations. Renal perfusion of the cortical compartment (CRBF) is known to be clearly higher compared to one of the medullary compartment (MRBF) [24, 25]. In our study the difference between CRBF and MRBF is relatively small, which may be explained by our analysis strategy not to include parts of the medullary in the cortical compartment during the segmentation processes. This leads to the fact that some of the actually cortical areas were evaluated as medullary tiate between individuals receiving a MRI scan for the first time from individuals with former experience of a MRI experience. An influence of stress due to the MRI scan on RBF in individuals having a MRI scan for the first time may be anticipated.

\section{Conclusion}

Our data suggest that the ASL-MRI technique is a promising method for the assessment of acute and shortterm changes of the total and cortical RBF. Most intriguing, due to the low variance of these RBF parameters, only small sample sizes are needed to detect clinically meaningful differences both between patient groups as well as after physiological or pharmacological interventions. From a clinical perspective, changes in renal perfusion might be detected in the early stage of a disease and thus may allow to detect patients at high risk for developing CKD. Protocol standardization for ASL-MRI is desperately needed, and large-scale prospective clinical trials with this MRI ASL technique should be initiated prior to their broad clinical use.

\section{Acknowledgements}

We gratefully acknowledge the expert technical assistance of Simone Pejkovic, Ulrike Heinritz, Dorothea Bader-Schmieder, Ingrid Fleischmann, Wiebke Maurer, Kerstin Fröhlich-Endreß, and Andrea Donhauser. respect to CRBF and MRBF. These associations with the perfusion. Another limitation is that we did not differen-

\section{Statement of Ethics}

Written informed consent was obtained from each patient before study inclusion. The study protocol of each trial was approved by the Local Ethics Committee (University of Erlangen-Nürnberg), and the studies were conducted in accordance with the Declaration of Helsinki and the principles of good clinical practice guidelines.

\section{Conflict of Interest Statement}

No conflict of interests.

\section{Funding Sources}

No funding received.

\section{Author Contributions}

D.K. designed the study, acquisition of data, analyzed all data, and wrote the manuscript. R.J. analyzed ASL-MRI data, contributed to methods and discussion, and reviewed the manuscript. A.B. contributed to acquisition of data and discussion and reviewed/edited the manuscript. S.J. contributed to acquisition of data and discussion and reviewed the manuscript. J.K. contributed to acquisition of data and discussion and reviewed the manuscript. K.S. contributed to acquisition of data and discussion and reviewed the manuscript. C.O. contributed to acquisition of data and discussion and reviewed the manuscript. P.M. MRI sequence developer, contributed to discussion, and reviewed the manuscript. M.S. contributed to discussion and reviewed the manuscript critically. M.U. contributed to discussion and reviewed the manuscript critically. R.E.S. designed the study, contributed to discussion, and reviewed the data/manuscript. All authors gave full consent for publication.

\section{References}

1 Jha V, Garcia-Garcia G, Iseki K, Li Z, Naicker S, Plattner B, et al. Chronic kidney disease: global dimension and perspectives. Lancet. 2013;382(9888):260-72.

2 James MT, Hemmelgarn BR, Tonelli M. Early recognition and prevention of chronic kidney disease. Lancet. 2010;375(9722):1296-309.

3 Herzog CA, Asinger RW, Berger AK, Chary$\tan$ DM, Díez J, Hart RG, et al. Cardiovascular disease in chronic kidney disease. A clinical update from kidney disease: improving global outcomes (KDIGO). Kidney Int. 2011;80(6): $572-86$.

4 Webster AC, Nagler EV, Morton RL, Masson P. Chronic kidney disease. Lancet. 2017; 389(10075):1238-52.

5 Inscho EW, Lewis K; Dahl Memorial Lecture. Lewis K. Dahl memorial lecture. Mysteries of renal autoregulation. Hypertension. 2009; 53(2):299-306. DOI: $10.1159 / 000513665$
Kannenkeril et al. 
6 Just A. Mechanisms of renal blood flow autoregulation: dynamics and contributions. Am J Physiol Regul Integr Comp Physiol. 2007; 292(1):R1-17.

7 Bidani AK, Griffin KA. Long-term renal consequences of hypertension for normal and diseased kidneys. Curr Opin Nephrol Hypertens. 2002;11(1):73-80.

8 Selby NM, Blankestijn PJ, Boor P, Combe C, Eckardt KU, Eikefjord E, et al. Magnetic resonance imaging biomarkers for chronic kidney disease: a position paper from the European Cooperation in Science and Technology Action PARENCHIMA. Nephrol Dial Transplant. 2018;33(Suppl_2):ii4-14.

9 Zhang JL, Morrell G, Rusinek H, Sigmund EE, Chandarana H, Lerman LO, et al. New magnetic resonance imaging methods in nephrology. Kidney Int. 2014;85(4):768-78.

10 Mancia G, Fagard R, Narkiewicz K, Redon J, Zanchetti A, Böhm M, et al. 2013 ESH/ESC practice guidelines for the management of arterial hypertension. Blood Press. 2014;23(1): 3-16.

11 Ritt M, Janka R, Schneider MP, Martirosian $\mathrm{P}$, Hornegger J, Bautz W, et al. Measurement of kidney perfusion by magnetic resonance imaging: comparison of MRI with arterial spin labeling to para-aminohippuric acid plasma clearance in male subjects with metabolic syndrome. Nephrol Dial Transplant. 2010;25(4):1126-33.
12 Hammon M, Janka R, Siegl C, Seuss H, Grosso R, Martirosian P, et al. Reproducibility of kidney perfusion measurements with arterial spin labeling at 1.5 tesla MRI combined with semiautomatic segmentation for differential cortical and medullary assessment. Medicine. 2016;95(11):e3083.

13 Lloyd S. Least squares quantization in PCM. IEEE Trans Inf Theory. 1982;28(2):129-37.

14 Van Acker BA, Koomen GC, Arisz L. Drawbacks of the constant-infusion technique for measurement of renal function. Am J Physiol. 1995;268(4 Pt 2):F543-52.

15 Schoenberg SO, Aumann S, Just A, Bock M, Knopp MV, Johansson LO, et al. Quantification of renal perfusion abnormalities using an intravascular contrast agent (part 2): results in animals and humans with renal artery stenosis. Magn Reson Med. 2003;49(2):288-98.

16 Fisher JP, Paton JF. The sympathetic nervous system and blood pressure in humans: implications for hypertension. J Hum Hypertens. 2012;26(8):463-75.

17 Haddock BT, Francis ST, Larsson HBW, Andersen UB. Assessment of perfusion and oxygenation of the human renal cortex and medulla by quantitative MRI during handgrip exercise. J Am Soc Nephrol. 2018;29(10): $2510-7$.

18 Leonard BL, Malpas SC, Denton KM, Madden AC, Evans RG. Differential control of intrarenal blood flow during reflex increases in sympathetic nerve activity. Am J Physiol Regul Integr Comp Physiol. 2001;280(1): R62-8.
19 Schneider MP, Janka R, Ziegler T, Raff U, Ritt $\mathrm{M}$, Ott C, et al. Reversibility of the effects of aliskiren in the renal versus systemic circulation. Clin J Am Soc Nephrol. 2012;7(2):25864.

20 Ott C, Bosch A, Winzer N, Friedrich S, Schinzel R, Tegtmeier F, et al. Effects of the nitric oxide synthase inhibitor ronopterin (VAS203) on renal function in healthy volunteers. Br J Clin Pharmacol. 2019;85(5):900-7.

21 Delles C, Klingbeil AU, Schneider MP, Handrock R, Schäufele T, Schmieder RE. The role of nitric oxide in the regulation of glomerular haemodynamics in humans. Nephrol Dial Transplant. 2004;19(6):1392-7.

22 Bjornstad P, Lovshin JA, Lytvyn Y, Boulet G, Lovblom LE, Alhuzaim ON, et al. Adiposity impacts intrarenal hemodynamic function in adults with long- standing type 1 diabetes with and without diabetic nephropathy: results from the Canadian Study of longevity in type 1 diabetes. Diabetes Care. 2018;41(4):831-9.

23 Schmieder RE, Beil AH, Weihprecht H, Messerli FH. How should renal hemodynamic data be indexed in obesity? J Am Soc Nephrol. 1995;5(9):1709-13

24 Martirosian P, Klose U, Mader I, Schick F. FAIR true-FISP perfusion imaging of the kidneys. Magn Reson Med. 2004;51(2):353-61.

25 Fenchel M, Martirosian P, Langanke J, Giersch J, Miller S, Stauder NI, et al. Perfusion MR imaging with FAIR true FISP spin labeling in patients with and without renal artery stenosis: initial experience. Radiology. 2006; 238(3):1013-21. 